\title{
ASYMPTOTIC SOLUTION OF A LAMINAR FLOW \\ IN A POROUS CHANNEL WITH LARGE SUCTION: A NONLINEAR TURNING POINT PROBLEM
}

\section{A. Dean MacGillivray and Chunqing Lu}

\begin{abstract}
The boundary-value problem

$$
\epsilon f^{(i v)}=\left[f f^{\prime \prime \prime}-f^{\prime} f^{\prime \prime}\right], \quad f(0)=f^{\prime \prime}(0)=f^{\prime}(1)=0, \quad f(1)=1,
$$

is a version of Berman's problem and its solutions describe a laminar flow in a channel with porous walls. When $0<\epsilon \ll 1$, the problem is a singular perturbation problem and physically corresponds to large suction through the walls. This case is known to possess three solutions, types I, II, III. The asymptotic analysis of the type III solution-the only nonmonotone solution for small positive $\epsilon$-is the subject of the present investigation. A feature of the analysis is the introduction of a transition layer approximation whose domain of uniform validity overlaps with the outer and the boundary-layer approximations. The asymptotic approximations are consistent with two theorems proved, and the validity of the asymptotics is strongly supported by evidence from numerical experiments.
\end{abstract}

\section{Introduction}

In this paper, we find the asymptotic behavior of type III solutions [13], [22] of one of Berman's problems. This has been an open problem in asymptotic analysis for many years.

Berman, in his investigation of the flow of an incompressible viscous fluid along a channel with parallel rigid porous walls [3], discovered a time independent similarity solution for the stream function. His procedure can be outlined briefly as follows. Assume the geometry of the channel is given as $y \in[-h, h], x \in(-\infty, \infty)$, where $h$ is the half-width of the channel. Berman assumed the stream function $\psi$ could be written as

$$
\psi=[h U(0)-V x] f\left(\frac{y}{h}\right),
$$

where $U(0)$ is an arbitrary velocity at the station $x=0$. Let $\eta=y / h$. Then the velocity components are given by

$$
\begin{gathered}
u=\frac{1}{h}[h U(0)-V x] f^{\prime}(\eta), \\
v=V f(\eta) .
\end{gathered}
$$

Received July 29, 1993.

1991 Mathematics Subject Classification: 34B15, 34E05, 34E20.

Key words and phrases: singular perturbations, transition layer, laminar flow, exponential terms. 
Substitution into the Navier-Stokes equations yields a third order differential equation for $f$,

$$
f^{\prime \prime \prime}=R\left[f f^{\prime \prime}-\left(f^{\prime}\right)^{2}\right]+k,
$$

where $k$ is a constant, and $R=V h / \nu$ is the Reynolds number of the flow based upon the velocity $V$. Note that if $V<0$ (injection through the walls), then $R<0$, and if $V>0$ (suction through the walls), then $R>0$. As usual, $\nu$ denotes the kinematic viscosity.

The boundary conditions $u=0$ and $v= \pm V$ at $y= \pm h$ imply $f^{\prime}( \pm 1)=0$ and $f( \pm 1)= \pm 1$. Equation (1.3) and these boundary conditions admit symmetric and antisymmetric flows. If the flow is assumed symmetric, then $f$ is an odd function of $\eta$, so that $f(0)=f^{\prime \prime}(0)=0$. It is convenient to differentiate (1.3) once. When this is done, and the boundary conditions for a symmetric self-similar solution are imposed, the following boundary-value problem is obtained:

$$
\begin{gathered}
\epsilon f^{(i v)}=\left(f f^{\prime \prime \prime}-f^{\prime} f^{\prime \prime}\right), \quad 0<\eta<1, \\
f(0)=f^{\prime \prime}(0)=0, \\
f(1)=1, \quad f^{\prime}(1)=0,
\end{gathered}
$$

where $\epsilon=1 / R$. The asymptotic analyses of (1.4), (1.5), (1.6) for small values of $\epsilon$ are singular perturbation problems, [6], [7], [11], [18]. In particular, the case $0<\epsilon \ll 1$, which corresponds to large suction, (i.e., $R \gg 1$ ) leads to three such problems, one of which is the subject of this paper.

This problem and related problems have been studied by Berman [3], Proudman [12], Yuan and Finkelstein [21], Terrill [16], Terrill and Thomas [17], Robinson [13], Skalak and Wang [15], and Zaturska et al. [22]. The importance of exponentially small terms in some of the analyses was commented on by Van Dyke [18].

The boundary-value problem (1.4-1.6) admits three distinct solution branches, originally found by Robinson [13] using numerical experimentation. He classified them as type I, type II, and type III solutions. Because the present paper is concerned only with the type III solution, we give only a brief summary of type I and type II solutions.

Type I and type II solutions. Type I solutions exist for all positive and negative values of $R$. Type II solutions do not exist below a certain critical positive value for $R$. Workers in this area seem agreed that this critical value, as determined by numerical experimentation, is close to 12.165 , and in fact type II and type III solutions emerge from the common solution that appears when $R \approx 12.165$. As $R$ increases without bound, solutions of type I and of type II become very similar, being separated over most of the interval $[0,1]$ by an exponentially small quantity. According to Robinson's asymptotic analysis, their common behavior is essentially linear, $f \sim \eta$ whereas, for large $R$, the solutions of type III first decrease from zero, reach a minimum, and then increase until $\eta=1$ is reached, where $f(1)=1$. See Figure 1.1 for the case $\epsilon=.01$.

Mathematical justification for the existence of solutions of type I was proved by Shih [14] using fixed point methods. More recently, the existence of all three types was proved by Hastings et al. [5]; see also proofs by Wang and Hwang [19]. A rigorous asymptotic analysis of solutions of type I for large negative $R$ appears in [8]. Included there are proofs of several conjectures based on numerical evidence obtained by Yuan and Finkelstein [21]. Recently, McLeod [10] has proved the validity of the asymptotic 


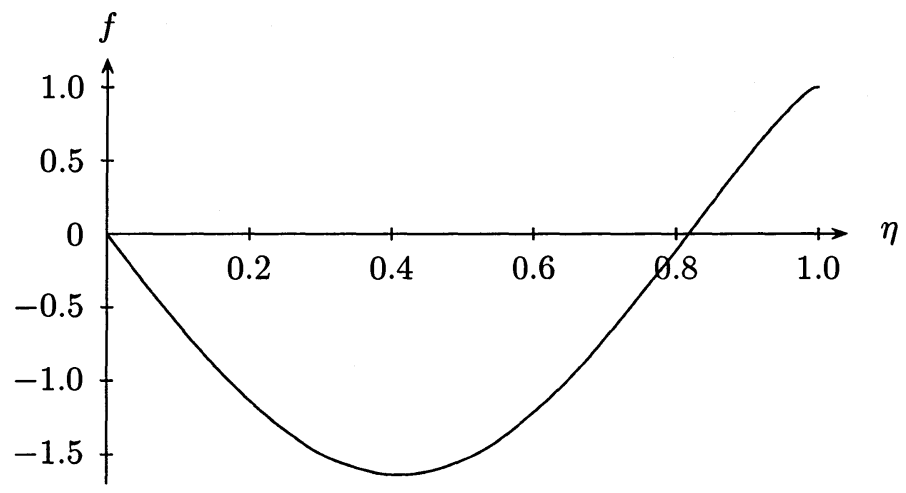

Figure 1. Plot of the type III solution of equations (1.4)-(1.6) for $\epsilon=.01$. The solution was obtained using the NDSolve command for Mathematica 2.1 for SPARC; for further information refer to Table 1 in Section 5. The relatively large value of $\epsilon$ was used in order to show the behavior as the boundary at $\eta=1$ is approached.

approximations for large $R$, previously obtained by Robinson for type I and type II flows.

Type III flows. We now turn our attention to the type III flows. A rigorous proof of the existence of all three types was given by Hastings et al. [5]. However, type III solutions have yet to be analyzed for $R \gg 1$, although efforts in this direction were made by Robinson [13] and by Zaturska et al. [22]. Both groups attempted to use the method of matched asymptotic expansions applied to (different) assumed outer and boundary-layer expansions. Certain constants which could not be determined by matching were estimated by a comparison with computer-generated solutions. Their resulting predictions are discussed in Section 5.

Robinson [13] apparently had some doubts about the validity of matching the outer approximation with a standard boundary-layer solution. We believe his comments show he clearly understood the subtle nature of the asymptotic problems involved. We shall introduce Robinson's insightful comments later.

Zaturska et al. [22], on the other hand, suggest their asymptotic analysis, if pushed to sufficiently high order, might determine the constants mentioned above, and their reliance on numerical curve fitting could be avoided. For a discussion about constants left undetermined by matching, see Bender and Orszag [2], especially p. 468 and problems 9.32, 9.33 on p. 482, and the important paper by Grasman and Matkowsky [4].

Our study of the type III solution, like those of Robinson and of Zaturska et al., is based on the method of matched asymptotic expansions. But in addition to the outer and boundary-layer approximations they considered, we have introduced a third approximation which has some similarities to the sort of transition approximation found, for example, in the study of the relaxation oscillations of Van der Pol's equation [6], in the sense that it provides a bridge between the outer and boundary-layer approximations. However, as we shall see, its analysis is quite unusual; specifically, new ideas about incorporating transcendental terms into the matching process are introduced. Nevertheless, we shall refer to it as the transition approximation.

Some of the ideas introduced in the present problem have found application in a completely unrelated area, namely nonlinear oscillation theory, [9].

The remainder of the paper is organized as follows. Section 2 contains two theorems 
and their proofs. Theorem 1 proves that if $R$ is sufficiently large, then the location of the single zero (the turning point referred to in the title) that exists for type III solutions occurs in the vicinity of $\eta=1$. Theorem 2 proves that the type III solutions and their second derivatives are unbounded on compact subintervals of $(0,1)$ as $R \rightarrow \infty$. Of course, any asymptotic analysis must conform to these properties. The formal part of the asymptotic analysis begins in Section 3, which is divided into two parts. The first part presents the analysis of the outer asymptotic approximation which is uniformly valid on closed subintervals of $[0,1)$. The second part of Section 3 presents the analysis of the boundary layer which is present at and in the vicinity of $\eta=1$. As outlined above, we supplement these with a third transition approximation. Since this transition approximation is the key to the success of our analysis, it is presented separately in Section 4. In Section 5, we compare our theory with numerical results using Mathematica 2.1. Confirming evidence using COLSYS has been provided by Robert Miura and Ray Spiteri. Mathematica is accessible to most readers, who can easily check our results and/or do some independent checks for themselves. Section 6 has some concluding remarks.

\section{Rigorous results}

There are several ways to argue that the internal zero (see Figure 1.1) of the type III solutions approaches $\eta=1$ as $\epsilon \downarrow 0$, including a reliance on computer-generated numerics [13], and numerics combined with physical arguments [22]. An heuristic argument based upon the original boundary-value problem also can be made. However, we needn't relay on any of these approaches, since this result is contained in Theorem 1 stated and proved below. We also prove, in Theorem 2, that the type III solutions tend to $-\infty$ as $R \rightarrow \infty$ on compact subintervals of $(0,1)$.

It is convenient to begin with a list of previously proved facts concerning type III solutions $f(\eta: \epsilon)$.

(a) $f^{(i v)}(\eta)<0$ on $(0,1]$; see [15] or [8, Lemma 1].

(b) Solutions with $f^{\prime}(0)<0$ exist for all sufficiently large $R$; [5].

(c) Properties (a) and (b), together with the original boundary conditions can be seen to imply that, for sufficiently large $R$, the graph of $f^{\prime \prime}$ first rises from zero, reaches a maximum, then crosses the $\eta$ axis at a point $y_{\epsilon}$ to become negative. See Figure 2.1 for a typical profile.

(d) Properties (a), (b), (c) imply $f(\eta)$ has a unique internal zero, $z_{\epsilon}$. It is proved in [5, Lemma 11] that $z_{\epsilon}<y_{\epsilon}$.

(e) Differentiating (1.4) gives $\epsilon f^{(v)}=f f^{(i v)}-f^{\prime 2}$, so that $f^{(v)}$ is negative for $\eta>z_{\epsilon}$.

Theorem 1. $\lim _{\epsilon \rightarrow 0+} z_{\epsilon}=\lim _{\epsilon \rightarrow 0+} y_{\epsilon}=1$, and $\lim _{\epsilon \rightarrow 0+} f^{\prime}\left(y_{\epsilon}\right)=\infty$.

The proof is an obvious consequence of Lemmas 1 and 2 below.

Lemma 1. $\lim _{\epsilon \rightarrow 0+}\left(y_{\epsilon}-z_{\epsilon}\right)=0$.

Proof. For contradiction, make the tentative assumption that there is an $\bar{a}>0$ and a decreasing sequence $\left\{\epsilon_{i}\right\}$ with limit 0 and $y_{\epsilon_{i}}-z_{\epsilon_{i}} \geq \bar{a}$. Sequential compactness of $[0,1]$ implies the existence of a subsequence, again denoted by $\left\{\epsilon_{i}\right\}$, such that $\left\{z_{\epsilon_{i}}\right\}$ and 


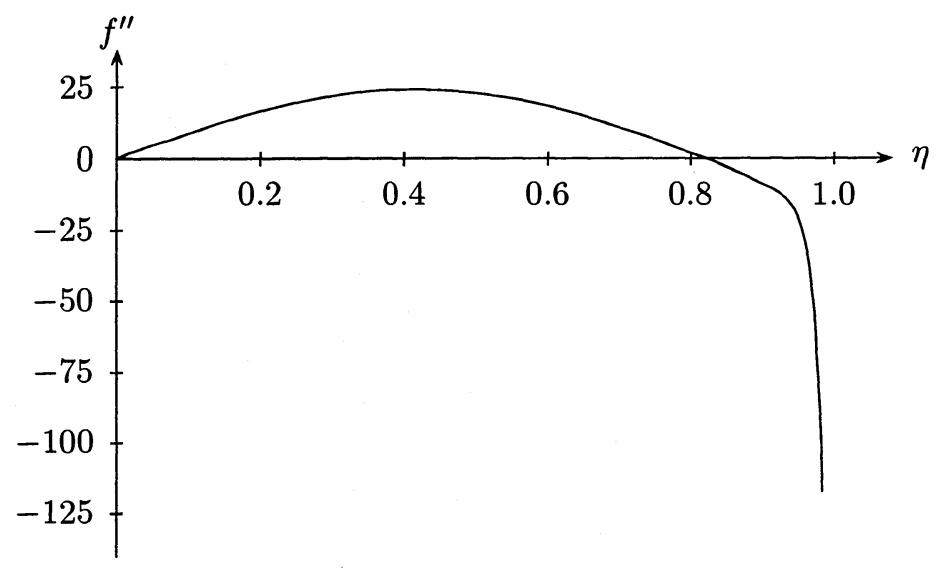

FIgURE 2. Plot of $f^{\prime \prime}(\eta)$. Note the concavity is everywhere down, a consequence of rigorous property (a).

$\left\{y_{\epsilon_{i}}\right\}$ converge to $z_{\infty}, y_{\infty}$, respectively, and $y_{\infty}-z_{\infty} \geq \bar{a}$. With no loss of generality, we can assume

$$
z_{\epsilon_{i}}<z_{\infty}+\frac{1}{6} \bar{a} \text { and } y_{\epsilon_{i}}>z_{\infty}+\frac{5}{6} \bar{a}
$$

Remark. For notational simplicity, we often write $f_{i}=f\left(\eta ; \epsilon_{i}\right), z_{i}=z_{\epsilon_{i}}$, and $y_{i}=y_{\epsilon_{i}}$.

The constant signs of $f_{i}, f_{i}{ }^{\prime}, f_{i}{ }^{\prime \prime}, f_{i}{ }^{(i v)}$, and $f_{i}{ }^{(v)}$ on the interval $\left[z_{\infty}+\bar{a} / 6, z_{\infty}+\right.$ $5 \bar{a} / 6]$ and the corresponding monotonicity of $f_{i}, f_{i}{ }^{\prime}, f_{i}{ }^{\prime \prime \prime}, f_{i}{ }^{(i v)}$ will be used to show that the sequences $\left\{f_{i}\right\},\left\{f_{i}{ }^{\prime}\right\},\left\{f_{i}{ }^{\prime \prime}\right\},\left\{f_{i}{ }^{\prime \prime \prime}\right\}$, and $\left\{f_{i}{ }^{(i v)}\right\}$ are all bounded on $\left[z_{\infty}+\right.$ $\left.\bar{a} / 3, z_{\infty}+4 \bar{a} / 9\right]$, so that the Arzela-Ascoli theorem can be used. The boundedness of $\left\{f_{i}\right\}$ is obvious since $f_{i}$ lies between 0 and 1 on $\left[z_{i}, 1\right]$. It is also easy to show $\left\{f_{i}^{\prime}\right\}$ and $\left\{f_{i}{ }^{\prime \prime}\right\}$ are bounded on $\left[z_{\infty}+\bar{a} / 6, z_{\infty}+2 \bar{a} / 3\right]$. For $\left\{f_{i}{ }^{\prime}\right\}$ this is obvious, since otherwise $\left\{f_{i}\right\}$ would become unbounded on the interval $\left[z_{\infty}+2 \bar{a} / 3, z_{\infty}+5 \bar{a} / 6\right]$, which is impossible. As for $\left\{f_{i}{ }^{\prime \prime}\right\}$, we note that because $f_{i}{ }^{\prime \prime}$ is positive and concave down on the interval, the area under the graph of $f_{i}{ }^{\prime \prime}$ exceeds the area of a triangle drawn with vertex at the maximum and base on the line segment joining $z_{\infty}+\bar{a} / 6$ and $z_{\infty}+2 \bar{a} / 3$; a large maximum would make $f_{i}{ }^{\prime}\left(z_{\infty}+2 \bar{a} / 3\right)$ large, which as we saw above is impossible.

We next note that $\left\{f_{i}^{\prime \prime \prime}\right\}$ is bounded on $\left[z_{\infty}+\bar{a} / 3, z_{\infty}+\bar{a} / 2\right]$, since otherwise $f_{i}^{\prime \prime \prime}\left(z_{\infty}+\bar{a} / 3\right) \rightarrow \infty$ for some subsequence of $\left\{\epsilon_{i}\right\}$ or $f_{i}^{\prime \prime \prime}\left(z_{\infty}+\bar{a} / 2\right) \rightarrow-\infty$ for some subsequence of $\left\{\epsilon_{i}\right\}$. In the former case, $f_{i}{ }^{\prime \prime}\left(z_{\infty}+\bar{a} / 3\right)-f_{i}{ }^{\prime \prime}\left(z_{\infty}+\bar{a} / 6\right)=\int_{z_{\infty}+\bar{a} / 6}^{z_{\infty}+\bar{a} / 3} f_{i}{ }^{\prime \prime \prime}$ will be unbounded. Since $f_{i}{ }^{\prime \prime}\left(z_{\infty}+\bar{a} / 6\right)$ is positive, $\left\{f_{i}{ }^{\prime \prime}\left(z_{\infty}+\bar{a} / 3\right)\right\}$ is forced to be unbounded, a possibility ruled out above. If, on the other hand, $f_{i}^{\prime \prime \prime}\left(z_{\infty}+\bar{a} / 2\right) \rightarrow$ $-\infty$ for some subsequence of $\left\{\epsilon_{i}\right\}$, an integration on $\left[z_{\infty}+\bar{a} / 2, z_{\infty}+2 \bar{a} / 3\right]$ leads to $\left\{f_{i}{ }^{\prime \prime}\left(z_{\infty}+\bar{a} / 2\right)\right\}$ being unbounded, a contradiction to the above proved fact that $\left\{f_{i}{ }^{\prime \prime}\right\}$ is bounded on $\left[z_{\infty}+\bar{a} / 6, z_{\infty}+2 \bar{a} / 3\right]$.

Finally, the sequence $\left\{f_{i}^{(i v)}\right\}$ must be uniformly bounded on $\left[z_{\infty}+1 / 3, z_{\infty}+4 \bar{a} / 9\right]$, since otherwise $f_{i}{ }^{(i v)}\left(z_{\infty}+4 \bar{a} / 9\right) \rightarrow-\infty$ for some subsequence $\left\{\epsilon_{i}\right\}$, and consequently 
(recall $f_{i}^{(i v)}$ is negative and decreasing)

$$
f_{i}^{\prime \prime \prime}\left(z_{\infty}+\bar{a} / 2\right)-{f_{i}}^{\prime \prime \prime}\left(z_{\infty}+4 \bar{a} / 9\right)=\int_{z_{\infty}+4 \bar{a} / 9}^{z_{\infty}+\bar{a} / 2} f_{i}^{(i v)}(\xi) d \xi
$$

approaches $-\infty$, so that, by taking subsequences, $f_{i}^{\prime \prime \prime}\left(z_{\infty}+\bar{a} / 2\right) \rightarrow-\infty$ or $f_{i}{ }^{\prime \prime \prime}\left(z_{\infty}+\right.$ $4 \bar{a} / 9) \rightarrow \infty$. In either case, the boundedness property of $f_{i}^{\prime \prime \prime}$ proved above is violated.

Applying the Arzela-Ascoli theorem, we see there is a subsequence $\left\{\epsilon_{i}\right\}$ such that the sequences $\left\{f_{i}\right\},\left\{f_{i}{ }^{\prime}\right\},\left\{f_{i}{ }^{\prime \prime}\right\},\left\{f_{i}{ }^{\prime \prime \prime}\right\}$ converge uniformly on $\left[z_{\infty}+\bar{a} / 3, z_{\infty}+4 \bar{a} / 9\right]$ to a function $h(\eta)$ and its first three derivatives, respectively, and furthermore, the boundedness property of $f_{i}^{(i v)}$ established above leads immediately to the conclusion that $h(\eta)$ satisfies the differential equation

$$
h h^{\prime \prime \prime}-h^{\prime} h^{\prime \prime}=0 \quad \text { on the interval } I \equiv\left[z_{\infty}+\bar{a} / 3, z_{\infty}+4 \bar{a} / 9\right] .
$$

It is quite easy to show that neither $h$ nor $h^{\prime \prime}$ can vanish on the closed interval $I$. For example, if $h$ vanished at some point $\eta_{0} \in I$, then for small $\epsilon_{i}, f_{i}$ is small on $\left[z_{i}, \eta_{0}\right]$, forcing $f_{i}{ }^{\prime}$ to be small and positive on $\left[z_{i}, z_{\infty}+\bar{a} / 4\right]$, say. This in turn means $f_{i}^{\prime \prime}$, being nonnegative and concave down on $\left[0, z_{\infty}+\bar{a} / 4\right]$, must be small at $z_{\infty}+\bar{a} / 4$. Since $f_{i}{ }^{\prime \prime}(0)=f_{i}{ }^{\prime \prime}\left(y_{i}\right)=0$, we now have three separated points where $f_{i}{ }^{\prime \prime} \geq 0$ is small. Together with the downward concavity of $f_{i}{ }^{\prime \prime}$, this means $f_{i}{ }^{\prime \prime}$ has a small upper bound on $[0,1]$. The same sort of circumstance is reached if $h^{\prime \prime}$ vanishes anywhere in $I$, since then, for large $i,\left|f_{i}{ }^{\prime \prime}\right|$ is small at three separated points, again leading to $f_{i}{ }^{\prime \prime}$ having a small upper bound on $[0,1]$. Thus, if either $h$ or $h^{\prime \prime}$ vanish on $I$, one can certainly make $f_{i}{ }^{\prime \prime}(\eta)<1$ on $[0,1]$ for all sufficiently large $i$. Integrate once:

$$
f_{i}{ }^{\prime}(\eta)=f_{i}{ }^{\prime}(0)+\int_{0}^{\eta} f_{i}{ }^{\prime \prime}(\xi) d \xi<\eta
$$

since $f_{i}^{\prime}(0)<0$. Integrating again, we obtain

$$
1=f_{i}(1)=\int_{0}^{1} f_{i}^{\prime}(\xi) d \xi<1,
$$

a contradiction. Thus, $h, h^{\prime \prime}$ cannot vanish on $I$.

Now that we know $h$ cannot be zero, we can use $(2.2)$ to find $\left(h^{\prime \prime} / h\right)^{\prime}$ :

$$
\frac{d}{d \eta}\left(\frac{h^{\prime \prime}}{h}\right)=\frac{h h^{\prime \prime \prime}-h^{\prime} h^{\prime \prime}}{h^{2}} \equiv 0
$$

Hence $h^{\prime \prime}=c h$, with $c \geq 0$. Immediately we find $h^{(i v)}=c^{2} h$. Now, if $c \neq 0, h^{\prime \prime}$ is concave up throughout $I$. But downward concavity (in the sense that the chord joining two points on a curve lies on or below the curve) is preserved in the limit; $h^{\prime \prime}$ cannot be concave up, and consequently $c=0$. Thus $h$ is linear and $h^{\prime \prime}$ is identically zero on $I$, contradicting our above result that $h^{\prime \prime}$ can vanish nowhere on $I$.

Thus our original tentative assumption that $y_{\epsilon}-z_{\epsilon}$ did not approach zero as $\epsilon \downarrow 0$ fails, and the proof of Lemma 1 is complete.

Lemma 2. $y_{\epsilon} \rightarrow 1$ as $\epsilon \downarrow 0$. 
Proof. If the lemma is false, then there is a sequence $\left\{\epsilon_{i}\right\}, \epsilon_{i} \downarrow 0$, such that $y_{i} \rightarrow y_{\infty}<1$; this uses the sequential compactness of $[0,1]$. Introduce points $a, b, c, d, e$, such that $y_{\infty}<a<b<c<d<e<1$. Without loss of generality, we can assume $y_{i}<a$. We first note that $f_{i}, f_{i}{ }^{\prime}$ are positive on $[a, 1]$ and $f_{i}{ }^{\prime \prime}, f_{i}{ }^{\prime \prime \prime}, f_{i}{ }^{(i v)}$ are negative on $[a, 1]$. From this, if $\left\{f_{i}{ }^{(i v)}\right\}$ were unbounded on $[a, c]$, then an integration on $[c, d]$ would make $\left\{f_{i}{ }^{\prime \prime \prime}\right\}$ unbounded on $[a, d]$; if this were true, then an integration on $[d, e]$ would make $\left\{f_{i}{ }^{\prime \prime}\right\}$ unbounded on $[a, e]$. Were this true, an integration on $[e, 1]$ would make $\left\{f_{i}^{\prime}\right\}$ become unbounded on $[b, 1]$, which in turn would make $\left\{f_{i}\right\}$ become unbounded on $[a, 1]$, a contradiction. Therefore, $\left\{f_{i}\right\},\left\{{f_{i}}^{\prime}\right\},\left\{{f_{i}}^{\prime \prime}\right\},\left\{f_{i}{ }^{\prime \prime \prime}\right\}$, and $\left\{f_{i}{ }^{(i v)}\right\}$ are bounded on the common interval $[b, c]$.

Applying the Arzela-Ascoli theorem, we find the limit function $h$ satisfies the differential equation (2.2). $h$ cannot vanish on $[b, c]$ because if $h\left(\eta_{0}\right)=0$ for some $\eta_{0} \in[b, c]$, then $f_{i}\left(\eta_{0}\right)$ can be made small, forcing $f_{i}{ }^{\prime}$ to be small on $[a,(a+b) / 2]$, which in turn forces $\left|f_{i}^{\prime \prime}\right|$ to be small at (say) the midpoint $a+(b-a) / 4$. Thus $\left|f_{i}^{\prime \prime}\right|$ is small at three separated points, a circumstance ruled out in the proof of Lemma 1 . As before, we are led to $h^{\prime \prime}=c h$ and $h^{(i v)}=c^{2} h$, but now $c \leq 0$. Since the downward concavity of $f_{i}^{\prime \prime}$ is preserved in the limit and $h$ is positive, we see it is necessary that $c=0$. Thus $h^{\prime \prime} \equiv 0$ on $[b, c]$, and as seen above, this is a contradiction. The proof of Lemma 2 is now complete.

Corollary. $f^{\prime}\left(y_{\epsilon} ; \epsilon\right) \rightarrow \infty$ as $\epsilon \rightarrow 0$.

Proof. Since $f\left(z_{\epsilon} ; \epsilon\right)=0, f(1 ; \epsilon)=1$, and $z_{\epsilon} \rightarrow 1$, the Mean Value Theorem tells us the maximum positive value of the derivative must be unbounded. Since this maximum occurs at the inflection point $y_{\epsilon}$, the conclusion follows.

Theorem 2. (a) $\lim _{\epsilon \rightarrow 0} f^{\prime \prime}(\eta ; \epsilon)=\infty$ for any $\eta \in(0,1)$,

(b) $\lim _{\epsilon \rightarrow 0} f(\eta ; \epsilon)=-\infty$ for any $\eta \in(0,1)$.

Proof of part (a). Select any point $\eta_{1} \in(0,1)$ and choose $\epsilon$ small enough that $y_{\epsilon}>\eta_{1}$. Since $f^{\prime \prime}\left(\eta ; y_{\epsilon}\right)$ is concave down and $f^{\prime \prime}(0)=f^{\prime \prime}\left(y_{\epsilon}\right)=0$, we have $f^{\prime \prime \prime} \leq f^{\prime \prime}\left(\eta_{1}\right) / \eta_{1}$ on $\left(\eta_{1}, y_{\epsilon}\right)$, and hence

$$
f^{\prime \prime}(\eta) \leq \frac{f^{\prime \prime}\left(\eta_{1}\right) \eta}{\eta_{1}}
$$

for $\eta_{1} \leq \eta \leq y_{\epsilon}$. On $\left(0, \eta_{1}\right)$, we see $f^{\prime \prime \prime} \geq f^{\prime \prime}\left(\eta_{1}\right) /\left(\eta_{1}-y_{\epsilon}\right)$, and an integration from $\eta_{1}$ to $\eta<\eta_{1}$ gives

$$
f^{\prime \prime}(\eta) \leq \frac{f^{\prime \prime}\left(\eta_{1}\right)\left(y_{\epsilon}-\eta\right)}{y_{\epsilon}-\eta_{1}} .
$$

An integration of $f^{\prime \prime}(\eta)$ over $\left[0, y_{\epsilon}\right]$ with (2.4) and (2.5) yields

$$
f^{\prime}\left(y_{\epsilon}\right) \leq \frac{1}{2} f^{\prime \prime}\left(\eta_{1}\right)\left[\frac{\eta_{1}\left(2 y_{\epsilon}-\eta_{1}\right)}{y_{\epsilon}-\eta_{1}}+\frac{y_{\epsilon}{ }^{2}-\eta_{1}{ }^{2}}{\eta_{1}}\right]+f^{\prime}(0) .
$$

From the corollary, $f^{\prime}\left(y_{\epsilon} ; \epsilon\right) \rightarrow \infty$ as $\epsilon \rightarrow 0$. This, together with $y_{\epsilon} \rightarrow 1$ as $\epsilon \rightarrow 0$ and $f^{\prime}(0)<0$, implies $f^{\prime \prime}\left(\eta_{1}\right) \rightarrow \infty$ as $\epsilon \rightarrow 0$. This completes the proof of part (a). 
Proof of part (b). Now let $\eta_{0} \in(0,1)$ be given. We will show $\lim _{\epsilon \rightarrow 0} f\left(\eta_{0} ; \epsilon\right) \rightarrow-\infty$. Consider $f^{\prime \prime}$ on the interval $\left[\eta_{0} / 2,\left(1+\eta_{0}\right) / 2\right]$. From part $(\mathrm{a}), f^{\prime \prime}$ becomes large at the endpoints $\eta_{0} / 2$ and $\left(\eta_{0}+1\right) / 2$ as $\epsilon \rightarrow 0$. Its concavity implies $f^{\prime \prime}$ becomes large, as $\epsilon \rightarrow$ 0 , uniformly on $\left[\eta_{0} / 2,\left(\eta_{0}+1\right) / 2\right]$. An integration on this interval leads immediately to the conclusion that

$$
\max \left(-f^{\prime}\left(\eta_{0} / 2\right), f^{\prime}\left(\left(\eta_{0}+1\right) / 2\right)\right) \rightarrow \infty \text { as } \epsilon \rightarrow 0 .
$$

Since $f^{\prime}(\eta) \leq f^{\prime}\left(\eta_{0} / 2\right)$ for $\eta \in\left[0, \eta_{0} / 2\right]$ and $f^{\prime}(\eta) \geq f^{\prime}\left(\left(\eta_{0}+1\right) / 2\right)$ for $\eta \in$ $\left[\left(\eta_{0}+1\right) / 2, z_{\epsilon}\right]$, we see that $f\left(\eta_{0} / 2\right)<\eta_{0} / 2 \times f^{\prime}\left(\eta_{0} / 2\right)$ and $f\left(\left(\eta_{0}+1\right) / 2\right)<$ $-f^{\prime}\left(\left(\eta_{0}+1\right) / 2\right) \times\left(z_{\epsilon}-\left(\eta_{0}+1\right) / 2\right)$. Equation (2.7) then implies

$$
\min \left(f\left(\frac{\eta_{0}}{2}\right), f\left(\frac{\eta_{0}+1}{2}\right)\right) \rightarrow-\infty \quad \text { as } \epsilon \rightarrow 0
$$

and consequently $f\left(\eta_{0}\right) \rightarrow-\infty$ as $\epsilon \rightarrow 0$. This completes the proof of part (b) of Theorem 2.

\section{Asymptotic analysis of the outer and boundary-layer approximations}

In this section, we treat first the outer asymptotic approximation, then the boundarylayer asymptotic approximation. First some notation. Recall $f(\eta)$ vanishes for exactly one point $z_{\epsilon}$ in $(0,1)$. That is, $f\left(z_{\epsilon}\right)=0$. We also define $\Delta=1-z_{\epsilon}$. We refer to $z_{\epsilon}$ as the crossover point. Mathematically, it is the turning point for a nonlinear turning point problem. Note that the turning point $z_{\epsilon}$ is unknown à priori.

A. Outer approximation. If one formally sets $\epsilon=0$ in (1.4), the reduced equation

$$
f_{0} f_{0}^{\prime \prime \prime}-f_{0}^{\prime} f_{0}^{\prime \prime}=0
$$

is obtained. This equation and the two boundary conditions at $\eta=0$ are satisfied by linear functions, $C \sinh \kappa \eta$ and $C \sin \kappa \eta$, a result first discussed by Proudman [12]. For $f^{\prime}(0)<0$, we expect the outer solution to be valid at least until the turning point is approached, and this consideration eliminates the linear solution and the hyperbolic sine from contention, leaving an expression proportional to $\sin (\pi \eta /(1-\Delta))$. Of course, the constant of proportionality should be negative. For now, we write

$$
f \sim-\kappa \sin \frac{\pi \eta}{1-\Delta}, \quad \kappa>0,
$$

uniformly on compact subintervals of $[0,1-\Delta)$.

B. Boundary-layer approximation. We know from Section 2 that $\Delta \rightarrow 0$ as $\epsilon \rightarrow 0$. Conceivably $\Delta=O(\epsilon)$, but the numerical evidence says not, and, in fact, convincingly tells us that $\Delta \gg \epsilon$. Consider Figure 1.1. There the boundary layer thickness is $\sim .01, \Delta \approx 0.2$. The situation in Figure 1.1 is typical, as the reader can verify, and so we adopt the working assumption, subject to confirmation at the conclusion of the asymptotic analysis, that

$$
\epsilon \ll \Delta \ll 1 .
$$

(The actual asymptotic behavior of $\Delta$ will be determined by matching, and its leading behavior turns out to be $O(\epsilon|\log \epsilon|)$.) Since $f$ must rise from zero to unity in this distance $\Delta$, one might expect $d f / d \eta$ to be $O(1 / \Delta)=o(1 / \epsilon)$, and this expectation is 
convincingly supported by computer experiments. Of course, $f \sim 1$ in the boundarylayer region. Introducing the boundary-layer variable $x^{*}$ defined by

$$
\epsilon x^{*}=1-\eta
$$

the differential equation (1.4) becomes

$$
\frac{d^{4} f}{d x^{*^{4}}}=-\left(f \frac{d^{3} f}{d x^{*^{3}}}-\frac{d f}{d x^{*}} \frac{d^{2} f}{d x^{*^{2}}}\right) .
$$

Again, $f \sim 1$ in the region of interest, so we write

$$
f\left(x^{*}\right) \sim 1+a f_{1}\left(x^{*} ; \epsilon\right)+\cdots, \quad|a| \ll 1 .
$$

Assuming in addition that in the region of interest $d f / d \eta=o(1 / \epsilon)$ or equivalently, $d f / d x^{*}=o(1)$, substitution into (3.4) yields

$$
f\left(x^{*}\right)=1+a\left(1-x^{*}-e^{-x^{*}}\right)+\cdots,
$$

where $a=a(\epsilon)$ is a constant yet to be found, but in any case satisfies $|a| \ll 1$ as $\epsilon \rightarrow 0$. (It turns out that

$$
a=\frac{\epsilon}{\Delta}
$$

but although this result is simple, its justification is quite subtle, as we shall see in the next section.)

\section{Transition layer asymptotics; exponential terms}

The transition approximation is to be valid on some interval containing the turning point $\eta=z_{\epsilon} \equiv 1-\Delta$. One of the major difficulties in deriving the transition approximation is the fact that the leading term must be a solution of the full original equation (1.4). See, for example, a problem treated by Kevorkian and Cole [6]; see especially their equations (2.5.16), (2.5.25), and their Figure (2.5.3). On this point, they make the extremely important observation: the exact solution of the full differential equation need not satisfy the boundary conditions-(1.5), (1.6) in the present case. In particular, any linear function is an exact solution of (1.4). (Computer experiments suggest the solutions behaves linearly from the vicinity of the crossover point up to the beginning of the boundary layer, where $f$ is close to unity.)

To study the transition approximation, we introduce the independent variable

$$
\tau=\frac{\eta-z_{\epsilon}}{\Delta}
$$

When (1.4) is written in terms of $\tau$, the result is

$$
\frac{\epsilon}{\Delta} \frac{d^{4} f}{d \tau^{4}}=\left(f \frac{d^{3} f}{d \tau^{3}}-\frac{d f}{d \tau} \frac{d^{2} f}{d \tau^{2}}\right) .
$$

The assumption that the leading term of the transition expansion is linear gives

$$
f=\gamma \tau+\cdots
$$

An attempt to match the leading term $\gamma \tau$ with the boundary-layer solution (3.6) is immediately seen to be successful because the exponential decay in (3.6) leaves us with a linear approximation that can be made to agree with the linear solution of (4.2) in 
the vicinity where $f \sim 1$, yet outside the boundary layer of thickness $O(\epsilon)$. Since $\Delta \gg \epsilon$, this leads to

$$
\gamma=1
$$

and so

$$
f \sim \tau \text {. }
$$

Matching this linear behavior with that of (3.6), we obtain $a=\frac{\epsilon}{\Delta}$ when we write (4.3) in $x^{*}$ variables. That is, the boundary-layer solution (3.6) becomes

$$
f \sim 1+\frac{\epsilon}{\Delta}\left(1-x^{*}-e^{-x^{*}}\right)+\cdots .
$$

Remark. This seems a good place to insert some important comments made by Robinson [13]:

An investigation of the numerical results for large $R$ indicates that none of the individual terms of $(2.7 \mathrm{c})$ are completely dominant throughout the viscous layer. Thus no simplification of the equation, that would be valid throughout the layer, could be made and the full equation would have to be solved [our italics].

Robinson had great insight and clearly understood the delicate nature of the asymptotics involved.

The transition asymptotic approximation we have thus far reads

$$
f=\tau+k_{1}(\epsilon) \tilde{f}_{1}(\tau)+k_{2}(\epsilon) \tilde{f}_{2}(\tau)+\cdots,
$$

where $k_{1}=o(1), \quad k_{2}=o\left(k_{1}\right)$, and we now must find $\tilde{f}_{1}$ and $\tilde{f}_{2}$ as well as the gauge functions $k_{1}$ and $k_{2}$. Substitution into (4.2) leads to

$$
\frac{\epsilon}{\Delta} \frac{d^{4} \tilde{f}_{1}}{d \tau^{4}}=\tau \frac{d^{3} \tilde{f}_{1}}{d \tau^{3}}-\frac{d^{2} \tilde{f}_{1}}{d \tau^{2}}
$$

which is a second order linear equation for

$$
u=\frac{d^{2} \tilde{f}_{1}}{d \tau^{2}}
$$

and one solution is

$$
u \equiv \tau \text {. }
$$

A second solution is, as can be immediately verified:

$$
u_{2}=-e^{\frac{\Delta}{2 \epsilon} \tau^{2}}+\frac{\Delta}{\epsilon} \tau \int_{0}^{\tau} e^{\frac{\Delta}{2 \epsilon} s^{2}} d s
$$

Now, if $u_{2}$ were retained as part of $u$, matching with the outer expansions would be impossible for the following reasons: to be of the correct order, the coefficient would have to be exponentially small. But although the part of the solution that contains $u_{2}$ could grow to $O(1)$, the matching requires that the corresponding terms in the two expansions (the outer and the transition) have the same functional behavior on an overlap domain. The second solution $u_{2}$ has an exponential behavior, but the outer solution (or, rather, its second derivative) does not; therefore, $u_{2}$ cannot contribute to 
$d^{2} \tilde{f}_{1} / d \tau^{2}$. (This is an example of the advantage of using matching over "patching"; see Van Dyke [18, p. 81].)

Absorbing a multiplicative constant into $k_{1}$, integrating (4.7) and (4.8) twice, and recalling $\tilde{f}_{1}(0)=0$, we obtain

$$
\tilde{f}_{1}(\tau)=\frac{\tau^{3}}{6}+r_{1} \tau
$$

where $r_{1}$ is a constant.

We now substitute (4.5) into (4.2) using (4.10.) It turns out that to balance terms, one should set $k_{2}=k_{1}^{2}$. The result, after some algebra, is the following linear nonhomogeneous equation for $f_{2}$ :

$$
\frac{\epsilon}{\Delta} \frac{d^{4} \tilde{f}_{2}}{d \tau^{4}}=\tau \frac{d^{3} \tilde{f}_{2}}{d \tau^{3}}-\frac{d^{2} \tilde{f}_{2}}{d \tau^{2}}-\frac{\tau^{3}}{3} .
$$

Differentiating once, we obtain

$$
\frac{d^{5} \tilde{f}_{2}}{d \tau^{5}}-\frac{\Delta}{\epsilon} \tau \frac{d^{4} \tilde{f}_{2}}{d \tau^{4}}=-\frac{\Delta}{\epsilon} \tau^{2}
$$

which is a first order linear equation for $d^{4} \tilde{f}_{2} / d \tau^{4}$ with general solution

$$
\frac{d^{4} \tilde{f}_{2}}{d \tau^{4}}=-\frac{\Delta}{\epsilon} e^{\frac{\Delta}{2 \epsilon} \tau^{2}} \int_{0}^{\tau} s^{2} e^{-\frac{\Delta}{2 \epsilon} s^{2}} d s-B_{2} e^{\frac{\Delta}{2 \epsilon} \tau^{2}}
$$

where $B_{2}$ is a constant to be determined.

Obviously (4.13) has the potential to make the fourth derivative as well as the third, second, first, and zeroth derivatives become exponentially large if $|\tau|$ is bounded away from zero. However, and this is crucial, it is possible to choose $B_{2}$ to prevent such a blowup for negative $\tau$ where matching with (3.2) is to take place. To determine this value of $B_{2}$, we first find the asymptotic behavior of the integral in (4.13) for $\tau=-|\tau|$, $\tau$ bounded away from zero. A standard calculation yields

$$
\begin{aligned}
\int_{0}^{-|\tau|} s^{2} e^{-\frac{\Delta}{2 \epsilon} s^{2}} d s & \\
= & -\left(\frac{2 \epsilon}{\Delta}\right)^{3 / 2}\left[\frac{\sqrt{\pi}}{4}-\left(\frac{\sqrt{\frac{\Delta}{2 \epsilon}}|\tau|}{2} e^{-\frac{\Delta}{2 \epsilon}|\tau|^{2}}+\frac{1}{4 \sqrt{\frac{\Delta}{2 \epsilon}}|\tau|} e^{-\frac{\Delta}{2 \epsilon}|\tau|^{2}}+\cdots\right)\right] .
\end{aligned}
$$

Thus (4.13) becomes, for $\tau<0, \quad \tau$ bounded away from zero,

$$
\begin{gathered}
\frac{d^{4} \tilde{f}_{2}}{d \tau^{4}}=-\frac{\Delta}{\epsilon} e^{\frac{\Delta}{2 \epsilon} \tau^{2}}\left[-\left(\frac{2 \epsilon}{\Delta}\right)^{3 / 2}\left(\frac{\sqrt{\pi}}{4}-\frac{\sqrt{\frac{\Delta}{2 \epsilon}}|\tau|}{2} e^{-\frac{\Delta}{2 \epsilon}|\tau|^{2}}+\left(1+O\left(2 \epsilon / \Delta / \tau^{2}\right)\right)\right)\right] \\
-B_{2} e^{\frac{\Delta}{2 \epsilon} \tau^{2}}
\end{gathered}
$$


from which it is clear that those terms which blow up exponentially are neutralized by choosing

$$
B_{2}=\left(\frac{2 \epsilon}{\Delta}\right)^{1 / 2} \frac{\sqrt{\pi}}{2}
$$

leaving the simple result

$$
\frac{d^{4} \tilde{f}_{2}}{d \tau^{4}} \sim \tau+\cdots
$$

Antidifferentiating four times gives

$$
\tilde{f}_{2} \sim \frac{\tau^{5}}{120}+C_{1} \frac{\tau^{3}}{6}+C_{2} \frac{\tau^{2}}{2}+C_{3} \tau+C_{4},
$$

and so, from (4.3), (4.10), and the above expression,

$$
f=\tau+k_{1}\left(\frac{\tau^{3}}{6}+r_{1} \tau\right)+k_{1}^{2}\left(\frac{\tau^{5}}{120}+C_{1} \frac{\tau^{3}}{6}+C_{2} \frac{\tau^{2}}{2}+C_{3} \tau+C_{4}\right)+\cdots,
$$

valid for $\tau<0$ and $\tau$ bounded away from zero.

We are now ready to match the expression (4.18) with the outer solution (3.2) which we write using its Taylor series in powers of $\left(\eta-z_{\epsilon}\right)$ :

$$
\begin{aligned}
-\kappa \sin \left(\frac{\pi \eta}{1-\Delta}\right)= & \kappa \sin \left(\frac{\pi\left(\eta-z_{\epsilon}\right)}{1-\Delta}\right) \\
= & \frac{\kappa \pi\left(\eta-z_{\epsilon}\right)}{1-\Delta}-\frac{\kappa}{6}\left(\frac{\pi\left(\eta-z_{\epsilon}\right)}{1-\Delta}\right)^{3} \\
& +\frac{\kappa}{120}\left(\frac{\pi\left(\eta-z_{\epsilon}\right)}{1-\Delta}\right)^{5}+\cdots
\end{aligned}
$$

Now (4.19) is to be matched with (4.18), which, of course, means $\tau$ is negative and bounded away from zero and $\eta-z_{\epsilon}$ approaches zero as $\epsilon \rightarrow 0$. In order to facilitate the comparison, we rewrite (4.19) in terms of $\tau$ :

$$
-\kappa \sin \left(\frac{\pi \eta}{1-\Delta}\right)=\kappa\left(\frac{\pi \tau \Delta}{1-\Delta}\right)-\frac{\kappa}{6}\left(\frac{\pi \tau \Delta}{1-\Delta}\right)^{3}+\frac{\kappa}{120}\left(\frac{\pi \tau \Delta}{1-\Delta}\right)^{5}+\ldots
$$

Now we compare the terms in (4.18) with those in (4.20). We see immediately from the linear terms that $r_{1}=0$ and

$$
\kappa=\frac{1-\Delta}{\pi \Delta} .
$$

We can next match the leading cubic terms in (4.18), (4.20), or we could instead match the quintic terms. If we compare the former, we obtain immediately

$$
k_{1}=-\left(\frac{\pi \Delta}{1-\Delta}\right)^{2}
$$

If, on the other hand, we match the leading $\tau^{5}$ coefficients, that is, $(\pi \Delta / 1-\Delta)^{4} / 120$ from (4.18) and $k_{1}^{2} / 120$ from (4.20), we find

$$
k_{1}= \pm\left(\frac{\Delta \pi}{1-\Delta}\right)^{2}
$$


These two independent determinations of $k_{1}$ agree if the negative sign in (4.23) is selected.

Remark. The consistency of (4.22) and (4.23) is gratifying. If $k_{1}$ as determined by comparing the cubic terms did not agree with that given by matching the $\tau^{5}$ terms, then the entire analysis would be in doubt. Notice that the leading cubic terms in the transition expansion come from different terms, namely from $\tilde{f}_{1}$ and $\tilde{f}_{2}$, respectively.

We now turn our attention to the question of matching the transition expansion with the boundary-layer solution. At this point we know

boundary layer:

$$
f\left(x^{*}\right)=1+\frac{\epsilon}{\Delta}\left(1-x^{*}-e^{-x^{*}}\right)+\cdots
$$

transition:

$$
f(\tau)=\tau-\left(\frac{\pi \Delta}{1-\Delta}\right)^{2}\left(\frac{\tau^{3}}{6}\right)+\left(\frac{\pi \Delta}{1-\Delta}\right)^{4} \tilde{f}_{2}(\tau)+\cdots,
$$

where $\tilde{f}_{2}(\tau)$ is obtained by antidifferentiating (4.13) four times. Since our matching is to take place where $f \sim 1$, i.e., where $\tau \sim 1$, we need only the asymptotic behavior of (4.25) for $\tau \sim 1$. From (4.13) and (4.16), we have

$$
\begin{aligned}
\frac{d^{4} \tilde{f}_{2}}{d \tau^{4}} & =-\frac{\Delta}{\epsilon} e^{\frac{\Delta}{2 \epsilon} \tau^{2}} \int_{0}^{\tau} s^{2} e^{-\frac{\Delta}{2 \epsilon} s^{2}} d s-\left(\frac{2 \epsilon}{\Delta}\right)^{1 / 2} \frac{\sqrt{\pi}}{2} e^{\frac{\Delta}{2 \epsilon} \tau^{2}} \\
& =-\left(\frac{2 \epsilon}{\Delta}\right)^{1 / 2} \sqrt{\pi} e^{\frac{\Delta}{2 \epsilon} \tau^{2}}+\tau+\cdots
\end{aligned}
$$

Multiplying by $k_{1}^{2}=\left[(\Delta \pi /(1-\Delta)]^{4}\right.$ and noting only $\tilde{f}_{2}$ contributes to the fourth derivative, we find

$$
\frac{d^{4} f}{d \tau^{4}}=-\pi^{9 / 2}\left(\frac{\Delta^{7 / 2}}{(1-\Delta)^{4}}\right) \sqrt{2 \epsilon} e^{\frac{\Delta}{2 \epsilon} \tau^{2}}+\left(\frac{\Delta \pi}{1-\Delta}\right)^{4} \tau+\cdots
$$

We write this in terms of the boundary-layer variables by substituting

$$
\tau=1-\frac{\epsilon x^{*}}{\Delta}
$$

into (4.27), yielding:

$$
\begin{aligned}
\left(\frac{\Delta}{\epsilon}\right)^{4} \frac{d^{4} f}{d x^{* 4}}= & \pi^{9 / 2} \Delta^{4}\left(\frac{2 \epsilon}{\Delta}\right)^{1 / 2} e^{\frac{\Delta}{2 \epsilon}\left(1-\frac{\epsilon x^{*}}{\Delta}\right)^{2}}+\Delta^{4} \pi^{4}\left(1-\frac{\epsilon x^{*}}{\Delta}\right)+\cdots \\
=- & \pi^{9 / 2}\left(\frac{\Delta}{1-\Delta}\right)^{4}\left(\frac{2 \epsilon}{\Delta}\right)^{1 / 2} e^{\frac{\Delta}{2 \epsilon}} e^{\frac{\epsilon}{2 \Delta} x^{* 2}} e^{-x^{*}} \\
& +\left(\frac{\Delta}{1-\Delta}\right)^{4} \pi^{4}\left(1-\frac{\epsilon x^{*}}{\Delta}\right)+\cdots
\end{aligned}
$$


We compare this with the fourth derivative of the boundary-layer solution (4.4) after multiplying by $(\Delta / \epsilon)^{4}$ :

$$
-\left(\frac{\Delta}{\epsilon}\right)^{3} e^{-x^{*}}
$$

Comparison of (4.28) and (4.29) shows the overlap domain must be such that

$$
\frac{\epsilon}{2 \Delta} x^{* 2} \ll 1 \text { and } x^{*} \gg 1
$$

leaving constant coefficients of $e^{-x^{*}}$ in both expressions which, when equated, gives

$$
\pi^{9 / 2}\left(\frac{\Delta}{1-\Delta}\right)^{4}\left(\frac{2 \epsilon}{\Delta}\right)^{1 / 2} e^{\Delta / 2 \epsilon}=\left(\frac{\Delta}{\epsilon}\right)^{3} .
$$

Squaring both sides and rearranging, we find the transcendental equation which determines $\Delta$,

$$
\frac{(\Delta / \epsilon) e^{\Delta / \epsilon}}{(1-\Delta)^{8}}=\frac{1}{2 \pi(\pi \epsilon)^{8}} .
$$

This result is in a sense the culmination of our labors. The values of $\Delta$ which satisfy this transcendental equation determine all of the constants explicitly. That is, ours is an $a b$ initio theory, with no dependence on computer-generated solutions of (1.4) to compute undetermined constants, in contrast to the reliance on curve-fitting in the theory of Robinson and in the theory of Zaturska et al. With such explicit formulas at hand, we can compare our theory with computer experiments. We also can make the necessary check on our working assumption that $\Delta \gg \epsilon$. Indeed, (4.32) yields (after taking the logarithm of both sides, selecting the dominating term, and rearranging):

$$
\Delta(\epsilon) \sim-8 \epsilon \log \epsilon \quad \text { as } \quad \epsilon \rightarrow 0
$$

which shows $\Delta \gg \epsilon$.

Equation (4.33) gives us the asymptotic behavior of $\Delta$. We shall, however, use Mathematica's FindRoot command to find precise numerical values for $\Delta$, because we wish to see how well our theory agrees with computer experiments. This we do in the next section. Before leaving this section, let us add two more consistency checks to those already made. First, the crucial property of the fourth derivative is that it be negative; recall that this is a rigorous result. The transition asymptotics indeed give negative values for all $\tau$, including $\tau=0$. Secondly, from (3.2), (4.21), and (4.33), we see that $f$ approaches $-\infty$ as $\epsilon \rightarrow 0$, and $f^{\prime \prime}$ approaches $\infty$ as $\epsilon \rightarrow 0$, in agreement with Theorem 2 proved in Section 2.

\section{Comparison of theory with computer experiments}

We have used Mathematica, version 2.1 for SPARC. Robert Miura and Ray Spiteri have kindly supplied us with results obtained using the program COLDAE, which with its predecessor COLSYS was developed at the University of British Columbia by Ascher, Christiansen, and Russell [1].

We have organized our comparison of theory with computer experiments around three tables, which we now discuss. 
Discussion of Table 1. The command NDSolve of Mathematica solves an initialvalue problem. Our equation is fourth order, and so, in addition to the conditions $f(0)=0, f^{\prime \prime}(0)=0$, one must guess values for $f^{\prime}(0)$ and $f^{\prime \prime \prime}(0)$, with successful guesses being those which result in $f(1)$ being close to unity and $f^{\prime}(1)$ being close to zero. Columns 2 and 3 of Table 1 are values of successful guesses, with columns 4 and 5 showing the resulting values for $f(1)$, and $f^{\prime}(1)$. Columns 6 and 7 give values of $\Delta$, which, recall, is defined to be the distance from the single internal zero $z_{\epsilon}$ of the solution to the right hand boundary. Column 6 is the value determined using the computer, and was read off from the data of the numerical solution; the subscript MATH indicates this is a computer-determined value using Mathematica. Column 7 is the value of $\Delta$ obtained by numerically solving the asymptotic expression (4.32) produced by our asymptotic theory. The last column gives the percentage deviation of the entries in columns 6 and 7; it is a measure of agreement between theory and experiment. It can be seen that this percentage deviation diminishes in a very satisfying manner as $\epsilon$ approaches zero.

The numbers in the columns are obtained from the output, and, not surprisingly, more digits are displayed than are really meaningful. We feel a reasonable estimate of the number of digits that are actually significant is always at least three, and in most cases four or more. The main justification for this claim is that when one compares the output of Mathematica with that from COLSYS, the agreement is very good to three places in all cases, and often more. For instance, in one of the most difficult cases, with $\epsilon=.00002$, the $\Delta$ values agree to five figures. Data obtained by Miura and Spiteri includes error estimates which are not included in our tables; most of the time these estimates imply accuracy far beyond four digits for the COLSYS results. In any case, 3 or 4 digit accuracy is adequate to keep intact the numerical evidence supporting the validity of our asymptotic theory. For the purposes of our theory, the reader may simply ignore the extra digits. Evidence for this is seen by comparing the last column of Table 1 with the last column of Table 3 . The entries are remarkably similar and, most importantly, both confirm the validity of the asymptotic theory.

Table 1 does not include values of $\Delta$ from the theory of Zaturska et al. [22] because they do not explicitly provide a means for its theoretical determination. However, it is not unreasonable to assume one can use their equations (5.24) and (5.27) for this purpose, arriving at the problem of solving

$$
-1+\epsilon^{1 / 4} \pi(.0894)\left(-1+\frac{1-\eta}{\epsilon}+e^{-(1-\eta) / \epsilon}\right)=0
$$

for $\Delta_{\mathrm{ZDB}}=1-\eta$. At $\epsilon=.0003226$, which is in the middle of their curve fitting range, this gives the theoretical value of .00889318 , a deviation of over $40 \%$ from the computer experiment value. At other values of $\epsilon$, the results are far worse. If this is indeed an acceptable means of using their theory to predict $\Delta$, it strongly suggests their theory has some shortcomings, quite apart from the disagreement with computer experiments to be illustrated in Table 2. It should be emphasized that Zaturska et al. [22] do not provide us with a formula for $\Delta$. This is unfortunate because curve fitting becomes more convincing if parameters other than those used for the curve fitting itself agree with experiment.

Discussion of Table 2. Here we compare the theories of Robinson [13] and of Zaturska et al. [22] with computer experiments. Both use values of $f^{\prime}(0)$ at three values of $\epsilon$ computed using their computer and software to evaluate constants in the 


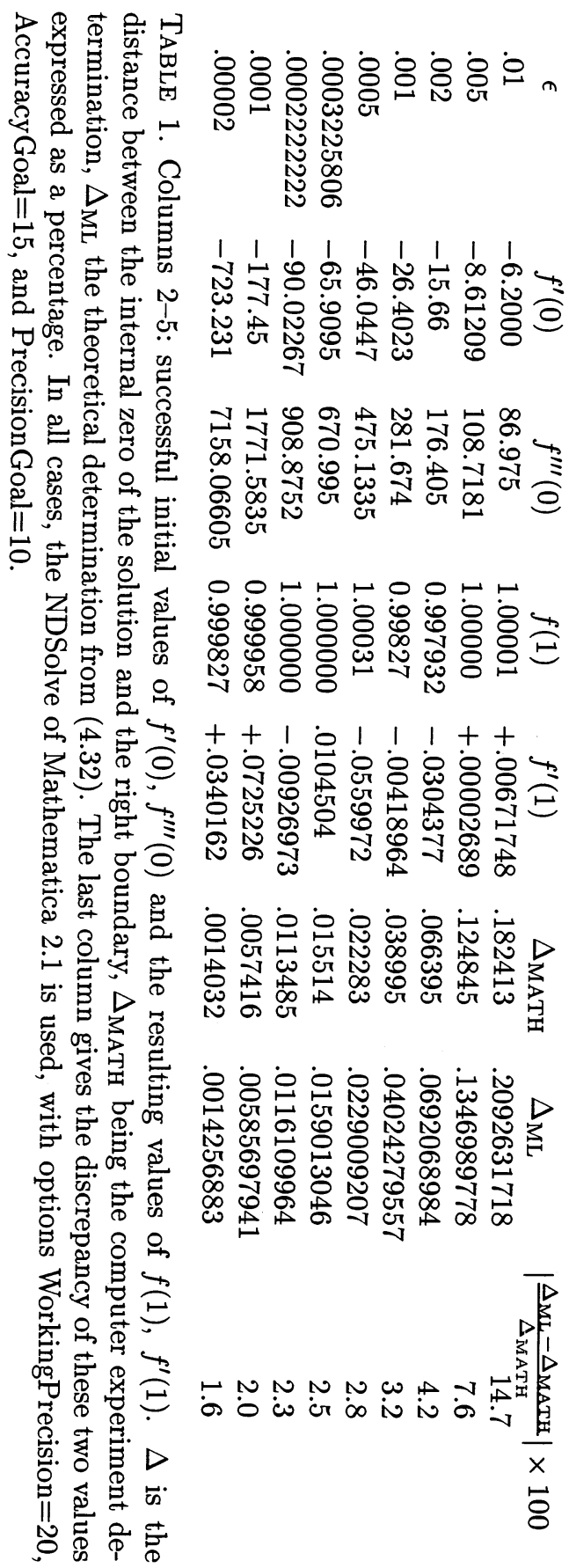


form of their assumed expansion. By definition, then, perfect agreement occurs at these selected values. What remains to be confirmed then is agreement at values of $\epsilon$ differing from these three values. Thus, column 2 repeats column 2 from Table 1. We infer from his paper that Robinson uses computer-generated values of $f^{\prime}(0)$ computed for $\epsilon=.002, .0018$, and .001 to compute the undetermined constants in his theory. Upon using these, his theoretical values must by definition agree perfectly with his computer solutions and be close to ours determined using Mathematica. His predictions for $f^{\prime}(0)$ are shown in column 3. Notice that outside the range of $\epsilon$-values used for the curve fitting, the predicted $f_{\mathrm{R}}^{\prime}(0)$ values swing away from the numerical values. For example, when $\epsilon=.0001$, the deviation (column 4 ) is about $17 \%$. At $\epsilon=.00002$, the deviation is $39 \%$. For some larger values of $\epsilon(\epsilon=.01$ and .005$)$, the table shows the deviation is large. In fact, the only values of $\epsilon$ where the deviation is small is in the range where the curve fitting took place.

For their curve-fitting, Zaturska et al. [22] use their computer-generated values of $f^{\prime}(0)$ at $R=2000,3100,4500$ which correspond to $\epsilon=.0005, .00032258, .000222 \ldots$ This means (by definition) perfect agreement with their computer results at these $\epsilon$-values, and one would, of course, expect very good agreement with our numerical results using Mathematica. And indeed, column 6 bears this out. However, as one moves outside the range of $\epsilon$-values used for the curve fitting, one finds the predicted values moving away from the Mathematica values. For instance, for $\epsilon=.0001$, there is almost a one percent discrepancy as compared with only .22 percent at $1 / 4500$, and at $\epsilon=.00002$, the discrepancy has already climbed to $6.6 \%$. Thus, as $\epsilon$ gets smaller, the error increases instead of diminishing as one would expect of a valid asymptotic theory.

Column 7 gives the predicted values of the present theory. We need not remind the reader that ours is an $a b$ initio theory, with no curve-fitting. Column 8 shows the deviation from the computer-determined values. The deviation steadily decreases from almost 23 percent when $\epsilon=.01$ to 3 percent when $\epsilon=.00002$.

Discussion of Table 3. Robert Miura and Ray Spiteri at the University of British Columbia have computed numerical solutions of the problem (1.4)-(1.6) using the program COLDAE, which is an advanced version of the familiar COLSYS [1]. Since in our asymptotic theory the determination of $\Delta$ is central, it is especially important in comparing our theory with computer experiments that the numerical values COLSYS gives for $\Delta$ are close to those obtained using Mathematica. The reader can readily see that this is indeed the case. Column 2 lists the values that COLSYS obtains for $f^{\prime}(0)$, and should be compared with the second column in Table 2 . Column 3 repeats our theoretical results from column 7 of Table 1 . Column 4 gives the values of $\Delta$ given by the COLSYS experiments of Miura and Spiteri. Column 5 gives the difference of the theoretical and experimental values of $\Delta$ (columns 3 and 4) expressed as a percentage. Once again, it is clear that the agreement is very good and improving (as it should) as $\epsilon$ decreases.

\section{Summary}

We have described the nonmonotone solution of one of Berman's problems (type III in Robinson's classification). We began by proving in Section 2 for $\epsilon \downarrow 0$ that the turning point at $z_{\epsilon}$ approaches 1 and that $f$ approaches $-\infty$ and $f^{\prime \prime}$ approaches $\infty$ on compact subintervals of $(0,1)$. Then we used formal matched asymptotic expansions to find, among other things, specific formulas for locating $z_{\epsilon}$ and for calculating $f$. 


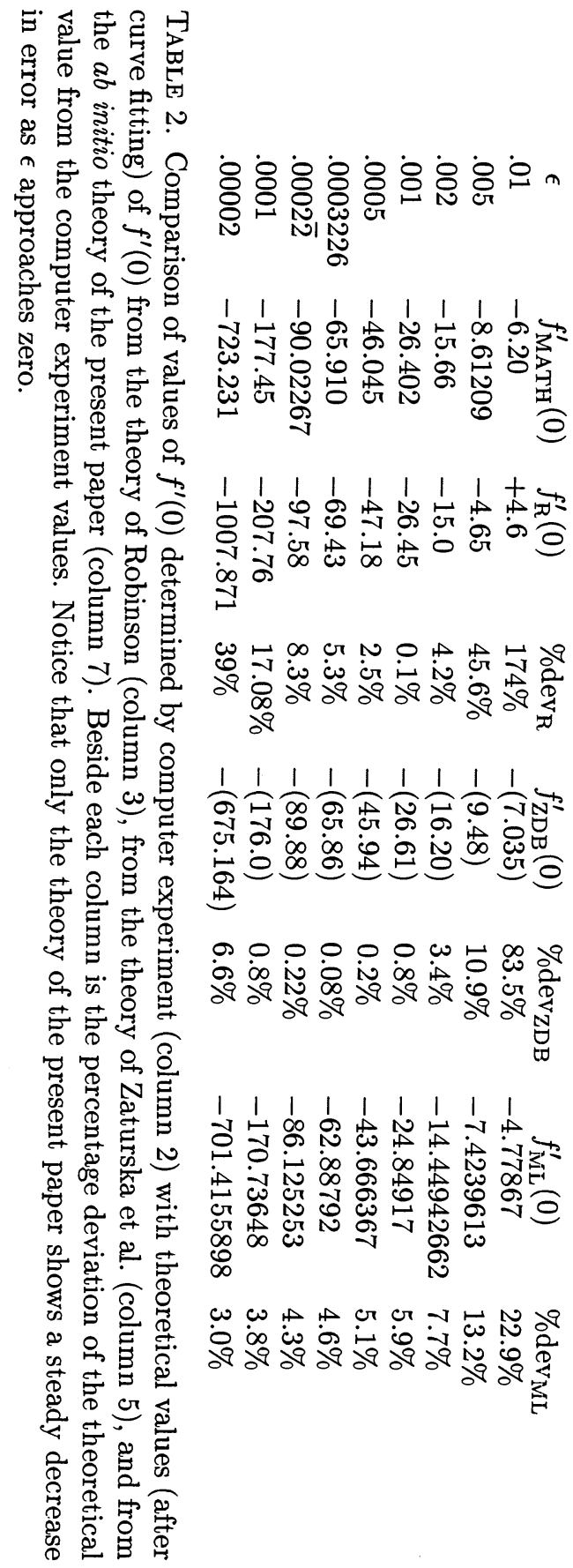




\begin{tabular}{llllc}
\multicolumn{1}{c}{$\epsilon$} & $f_{\text {COLSYS }}^{\prime}(0)$ & $\Delta_{\mathrm{ML}}$ & $\Delta_{\text {COLSYS }}$ & \%deviation \\
.01 & -6.19996 & .2092631718 & .182428 & 14.71 \\
.005 & -8.612086 & .134698778 & .1248447 & 7.89 \\
.002 & -15.71590 & .0692068984 & .06628865 & 4.40 \\
.001 & -26.48372 & .04024279557 & .03894195 & 3.34 \\
.0005 & -46.01893 & .0229009207 & .02228796 & 2.75 \\
.0003225806 & -65.95747 & .0159013046 & .01551393 & 2.49 \\
.0002222222 & -90.02266 & .0116109964 & .01134848 & 2.31 \\
.0001 & -177.4640 & .00585697941 & .005741415 & 2.01 \\
.00002 & -723.469 & .0014256883 & .0014032 & 1.60
\end{tabular}

TABLE 3. Results from computer experiments using COLSYS, courtesy Miura and Spiteri. Columns 2 and 4 show the values COLSYS gives for $f^{\prime}(0)$ and $\Delta$. Our theoretical values for $\Delta$ are rewritten in column 3 , and column 5 displays the discrepancy between the computer results in column 4 and the theoretical results in column 3 , expressed as a percentage. Compare with the last column in Table 1. The results support our asymptotic theory.

These asymptotic results are completely consistent with the rigorous results. Finally, we found that predictions based on our asymptotic theory compared very favorably with the results of computer experiments done by ourselves using Mathematica, and independently by Miura and Spiteri using COLSYS.

An interesting and, at the same time, essential feature of the asymptotic analysis is the introduction of a transition approximation which covers the region of the turning point. The construction of this intermediate expansion, and its matching with the outer and boundary-layer approximations, incorporates and exploits some ideas about exponential terms, which may have application to other problems and, in fact, have already been applied to one problem outside of fluid dynamics [9].

Previous attempts to obtain the asymptotic behavior of the type III solution were made by Robinson [13] and by Zaturska et al. [22]. In Section 5, we have compared their theories with computer experiments and conclude that the form of their results (which incidentally are different from each other) is not supported by the numerical evidence. Specifically, Robinson proposes $f^{\prime}(0) \sim \alpha R^{-1}$ and Zaturska et al. propose $f^{\prime}(0) \sim \beta R^{-3 / 4}$, where the constants are determined by computer. Our theory, whose results are supported by the computer experiments, shows the asymptotic dependence of $f^{\prime}(0)$ on $\epsilon=1 / R$ is not a simple power at all, but instead is given by $f^{\prime}(0) \sim$ $-1 / \Delta$, where $\Delta$ satisfies the transcendental equation (4.32). This surprising result was obtained from the careful study and analysis of certain exponential behavior properties in the transition approximation and the rational incorporation of this behavior into the matching principles of the method of matched asymptotic expansions.

Acknowledgements. We are happy to acknowledge many stimulating conversations with Professor Stuart Hastings. We wish also to thank Professor Robert Miura and Graduate Student Ray Spiteri at the University of British Columbia for supplying us with extensive numerical data obtained using COLSYS.

A. D. M. wishes to express his thanks to Professors N. Cernansky and J. Poole of Drexel University for their kind hospitality during his visit there. 


\section{References}

1. U. Ascher, R. Christiansen, and R. Russell. Collocation software for boundary value O. D. E. 's, Math. Comp. 33 (1979), 659-678.

2. C. A. Bender and S. Orszag, Advanced Mathematics for Scientists and Engineers, McGraw-Hill, New York (1978).

3. A. S. Berman, Laminar flow in channels with porous walls, J. Appl. Physics. 24 (1953), 12321235.

4. J. Grasman and B. J. Matkowsky, A variational approach to singularly perturbed boundary value problems for ordinary and partial differential equations with turning points, SIAM J. Appl. Math. 32 (1976), 588-597.

5. S. P. Hastings, C. Lu, and A. D. MacGillivray, A boundary value problem with multiple solutions from the theory of laminar flow, SIAM J. Math. Anal. 23 (1992), 201-208.

6. J. Kevorkian and J. D. Cole, Perturbation Methods in Applied Mathematics. Springer-Verlag, New York (1981).

7. P. A. Lagerstrom, Matched Asymptotic Expansions, Springer-Verlag, New York (1989).

8. C. Lu, A. D. MacGillivray, S. P. Hastings, Asymptotic behavior of solutions of a similarity equation for laminar flows in channels with porous walls, I.M.A. J. Applied Mathematics 49 (1992), 139-162.

9. A. D. MacGillivray, B. Liu, and N. D. Kazarinoff, Asymptotic analysis of the peeling-off point on a duck trajectory, To appear in this journal.

10. J. B. McLeod, Laminar flow in a porous channel, in Asymptotics Beyond All Orders, \#284 NATO ASI series, editors H. Segur, S. Tanveer, H. Levine. Plenum Press, New York (1991).

11. R. E. O'Malley, Introduction to Singular Perturbations, Academic Press, New York (1974).

12. I. Proudman, An example of steady laminar flow at large Reynolds number, J. Fluid Mech. 9 (1960), 593-602.

13. W. A. Robinson, The existence of multiple solutions for the laminar flow in a uniformly porous channel with suction at both walls, Journal of Engineering Mathematics 10 (1976), 23-40.

14. K. G. Shih, On the existence of solutions of an equation arising in the theory of laminar flow in a uniformly porous channel with injection, SIAM J. Appl. Math. 47 (1987), 526-533.

15. F. M. Skalak and C.Y. Wang, On the nonunique solutions of laminar flow through a porous tube or channel, SIAM J. Appl Math. 34 (1978), 535-544.

16. R. M. Terrill, On some exponentially small terms arising in flow through a porous pipe Quart. Journal of Mechanics and Applied Math. 26 (1973), 347-354.

17. R. M. Terrill and P.W.Thomas, On laminar flow through a uniformly porous pipe, Appl. Sci. Res. 21 (1969), 37-67.

18. M. D. Van Dyke, Perturbation Methods in Fluid Mechanics, Parabolic Press, Stanford (1975).

19. C. A. Wang and T.-W. Hwang, On multiple solutions of Berman's equation, Proc. Roy. Soc. Edinburgh, to appear (1991).

20. S. Wolfram, Mathematica, a System for Doing Mathematics by Computer, Second edition, Addison-Wesley, New York, (1991).

21. S. W. Yuan and A. B. Finkelstein, Laminar pipe flow with injection and suction through a porous wall, Trans. ASME, Ser.E, J. Applied Mech. 78 (1956), 719-724.

22. M. B. Zaturska, P. G. Drazin, and W. H. H. Banks, On the flow of a viscous fluid driven along a channel by suction at porous walls, Fluid Dynamics Research 4 (1988), 151-178.

Dept of Mathematics, State University of New York at Buffalo, Buffalo, New York

Department of Mathematics and Statistics, Southern Illinois University at Edwardsville, EDWARDSVILLE, ILLINOIS 